\title{
Phytoprotection
}

\section{Index des auteurs, volume 83}

Author Index, Volume 83

Volume 83, numéro 3, 2002

URI : https://id.erudit.org/iderudit/706240ar

DOI : https://doi.org/10.7202/706240ar

Aller au sommaire du numéro

\section{Éditeur(s)}

Société de protection des plantes du Québec (SPPQ)l

ISSN

0031-9511 (imprimé)

1710-1603 (numérique)

Découvrir la revue

Citer ce document

(2002). Index des auteurs, volume 83. Phytoprotection, 83(3), 169-170.

https://doi.org/10.7202/706240ar d'utilisation que vous pouvez consulter en ligne.

https://apropos.erudit.org/fr/usagers/politique-dutilisation/ 


\section{Index des auteurs, volume 83 Author Index, Volume 83}

\section{A-B}

Amri, A.

Anderson, T.R.

Angeli, G.

Arnaut, G.

Asselin, M.

Baidani, A.

Barasubiye, $T$.

Barnouin, T.

Bauce, É.

Beaulieu, C.

Beaulieu, J.

Bélair, G.

Bélanger, $A$.

Bélanger, $\mathrm{L}$.

Bélanger, R.R.

Béliveau, C.

Bellemare, J.

Bergeron, J.-M.

Bernier, L.

Berthiaume, R.

Bidon, Y.

Boerboom, C.M.

Boisclair, J.

Boiteau, G.

Boivin, $\mathrm{G}$.

Bostanian, N.J.

Bourgeois, G.

Breuil, C.

Brodeur, J.

Brosseau, M.

Brown, A.

Brunet, J.-F.

Buitenhuis, R.

\section{C-D}

Carignan, S.

Carisse, $\mathrm{O}$.

Caron, J.

Charbonneau, F.

Charest, M.

Chevrier, N.

Chouinard, G.

Christie, B.R.

Coderre, D.
$48,53,62$

158,165

48

$66,111,158,166$

$55,61,66$

47

73,165

47,59

158

58

$159,162,164,166$

\section{8}

48

99

48,49

49

$50,54,55,57,62,65$

51,158

51

162,166

66

61,66

52

52

52

68

1

73

48

62

159

$51,53,64$

41

$55,63,64$
Cormier, D. 49

Coulombe, C.

62

Cusson, M.

47,59

Dallaire, $R$.

Dangi, O.P.

Daniel, A.

Dauphinais, N.

Delisle, J.

Dewdney, M.

Dionne, J.

Dixon, P.L.

Drapeau, P.

Duke, G.M.

\section{F-H}

Fillmore, S.A.E.

Forti, D.

Fortier, M.

Fournier, F.

Fournier, $Y$.

Fradet-Turcotte, A.

Frigon, N.

Gagné, I.

Gagnon, M.

Gallant, C.E.

Gauvin, M.-J.

Gingras, D.

Girard, F.

Goettel, M.S.

Goldansaz, S.H.

Gonsard. P.-A.

Goulet, $\mathrm{H}$.

Grondin, J.

Guay, S.

Hamel, C.

Hébert, $C$.

Hillier, N.K.

Hogue, R.

\section{I-L}

Ioriatti, C.

Jabaji-Hare, S.

Jacob, $\mathrm{S}$.

Jeannotte, $R$.

Jones, M.W.
57

160, 166

57

41

57

54

62

111,158

165

158

55

48

41

55

68

56

89

56

68

158

159

159

160,166

$47,48,65$

139

160

160,166 
Juteau, M.

Kimpinski, J.

Kirouac, M.

Kovach, J.

Kushalappa, A.

Labonté, J.

Labrie, G.

Lafond, J.

Lajeunesse, J.

Laprade, R.

Lasnier, J.

Lavallée, $R$.

Laverdière, L.

Le Binh, T.

Lebel, G.

Lemieux, C.

Leroux, G.D.

Lesage, L.

Levasseur, A.

Lévesque, C.A.

Lhaloui, S.

Loppnau, P.

Lucas, É.

\section{M-O}

Marceau, L.

Marchand, D.

Marcotte, M.

Martinez, C.

Martinez, S.

Masson, L.

Mattedi, L.

Mauffette, Y.

McKeown, A.W.

McNeil, J.N.

McRae, K.B.

Mignault, M.-P.

Morin, C.

Morin,Y.

Musabyimana, $\mathrm{T}$.

Nakano, A.

Nicole, M.-C.

Noronha, C.

Nsarellah, $\mathrm{N}$.

Ouedraogo, R.M.

P-R

Pageau, D.

Palcic, M.M.

Panneton, B.

Papadopoulos, Y.A.
$52,56,60,63$
Pelletier, D.

62

Pelletier, F.

63

Pelletier, L.

63

Peyronnet, $\mathrm{O}$.

Plourde, K.

Potter, J.W.

Préfontaine, G.

Pronier, I.

Provost, C.

Racette, G.

Rancourt, B.

Rehse, $P$.

Renaud, J.-P.

Rioux, D.

Rioux, S.

Roy, M.

164

115,147

56,59

167

64

51

$68,164,167$

\section{S-Z}

Saint-Arnaud, M.

160,166

Saint-Germain, M. $\quad 65$

Saint-Louis, M. 65

Sanssouci, É. $\quad 165$

Schwart, J.-L. 52, 54, 56, 59

Shinners-Carnelley, T.C. 31

Simard, J.-N.

Simard, L.

66,166

Sommerville, D.W.

166

Stoltenberg, D.E. $\quad 99$

Stoltz, D. $\quad 59$

$\mathrm{Su}, \mathrm{Y}$. $\quad 56$

Szpacenko, A. 31

Tabone, É. $\quad 68$

Tanguay, $P$. 162, 166

Tenuta, A. $\quad 157$

Tewari, J.P. $\quad 31$

Thibodeau, P.O. $\quad 73$

Tremblay, É. $\quad 66$

Trudeau, M. 51, 158

Tweddell, R.J. 165

Vachon, V. 52, 54, 56, 59

Van Rie, J. $\quad 52$

Vanasse, A. $\quad 160$

Vanoosthuyse, F. $\quad 66$

VanRietschoten, S.E. $\quad 166$

Villeneuve, C. $\quad 67$

Vincent, C. $\quad 67,68,158,164,167$

Volenberg, D.S. $\quad 99$

Vujanovic, V. 160, 166

Wajnberg, É. $\quad 68$

Wyman, J.A. $\quad 69$

Yiğit, F. $\quad 125$

Zeybek, A. $\quad 125$ 